\title{
Formación de maclas durante el enfriamiento en aceros inoxidables superferríticos envejecidos
}

\author{
M.N. Salán* y M.J. Anglada*
}

\begin{abstract}
Resumen Los aceros inoxidables superferríticos se caracterizan por su estructura cúbica centrada, libre de austenita a cualquier temperatura. Sometidos a temperaturas intermedias, próximas a $475^{\circ} \mathrm{C}$, revelan una modulación del contenido en $\mathrm{Cr}$ de la red. Después de tiempos prolongados se forma fase $\alpha$, fragilizante y rica en cromo, fenómeno conocido como fragilización a $475^{\circ} \mathrm{C}$. En este trabajo, los tratamientos de envejecimiento realizados a temperaturas intermedias para un superferrítico DIN 1.4575 han permitido relacionar el incremento de dureza asociado a éstos con el tiempo de tratamiento, mediante una expresión coherente con un proceso térmicamente activado. Por otra parte, se ha detectado la presencia de maclas en el acero envejecido y templado en agua, siendo proporcional su densidad al grado de endurecimiento provocado por el tratamiento.
\end{abstract}

Palabras clave Superferríticos. Envejecimiento térmico. Fragilización. Maclado.

\section{Twinning induced by quenching in aged superferritic stainless steels}

\begin{abstract}
Superferritic stainless steels show a BCC structure free of austenite at any temperature. Intermediate ageing temperatures, close to $475{ }^{\circ} \mathrm{C}$, induce a-chromium content modulation and only after long periods of time, it is possible to detect chromium rich a' phase, which is responsible for embrittlement $\left(475^{\circ} \mathrm{C}\right.$ embrittlement). In this work, ageing thermal treatments at intermediate temperatures in the superferritic stainless steel DIN 1.4575 , have allowed to relate the associated hardness increase with time and temperature by means of a relationship that is compatible with a thermally activated process. After quenching from ageing temperature, twinning formation has been observed, and the extent of twinning is proportional to the increase in hardness, showing in this way that twinning density is proportional to embrittlement level.
\end{abstract}

Keywords Superferritic. Thermal ageing. Embrittlement. Twinning.

\section{INTRODUCCIÓN}

Los aceros inoxidables superferríticos son aleaciones hierro-cromo-carbono con pequeñas adiciones de otros elementos, su estructura es ferrítica a cualquier temperatura, son magnéticos y no son susceptibles de transformación, de manera que no es posible mejorar sus características mecánicas mediante tratamientos térmicos si bien, el trabajo en frío puede aumentar el límite elástico (acritud). Presentan contenidos muy bajos en intersticiales $(\mathrm{C}+\mathrm{N}<0,05 \%)$ y, también, porcentajes elevados de $\mathrm{Cr}(>22 \%)$, los cuales contribuyen a estabilizar la estructura ferrítica.
La permanencia prolongada a temperaturas intermedias, por debajo de $550{ }^{\circ} \mathrm{C}$, conduce a la formación de una fase rica en cromo, $\alpha$, que provoca una importante disminución en la energía absorbida a impacto. Este efecto fragilizante es tanto más notable cuanto mayor es el contenido en cromo y

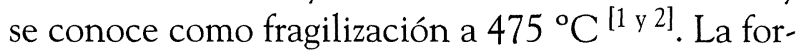
mación de fase $\alpha$ ' se inicia mediante fluctuaciones en la concentración de cromo según un mecanismo de descomposición espinodal de la ferrita $\left(\alpha \rightarrow \alpha^{\prime}\right)$, fenómeno gobernado por la difusión de cromo en la ferrita y que, por tanto, es térmicamente activado ${ }^{[1-4]}$, por lo cual la cinética de envejecimiento, en principio, podría describirse a partir de una ley de Arrhenius ${ }^{[5}$ y 6$]$.

(*) Departamento de Ciencia de los Materiales e Ingeniería Metalúrgica. Universitat Politècnica de Catalunya. Tel.: +34934016701 / +34934054454. FAX: +34934016706. nuria.salan@upc.edu 
Formación de maclas durante el enfriamiento en aceros inoxidables superferríticos envejecidos M.N. SALÁN Y M.J. ANGLADA

\section{MATERIALES Y PROCEDIMIENTO EXPERIMEN- TAL}

El material objeto de este trabajo ha sido un acero inoxidable superferrítico DIN 1.4575, suministrado por Thysen A.G. en estado de recocido tras un laminado en caliente, cuya composición nominal se indica en la tabla I.

El estudio metalográfico realizado con el material en estado de recepción, revela una distribución heterogénea de granos equiaxiales (Fig. 1). El tamaño de grano se ha determinado en $113 \pm 71 \mu \mathrm{m}$, que equivale a un tamaño 3.6 según norma ASTM 112.

Se han realizado tratamientos térmicos de envejecimiento a temperaturas comprendidas dentro de un rango intermedio, en hornos eléctricos y en ambiente de laboratorio. Las condiciones de envejecimiento consideradas se recogen en la tabla II. En todos los casos, el enfriamiento del material envejecido se ha realizado por inmersión en agua desde la temperatura de tratamiento.

La evaluación del efecto de los tratamientos térmicos de envejecimiento en las propiedades mecánicas del material se ha realizado a partir de medidas de dureza Vickers con cargas aplicadas de 1 $\mathrm{kg}$. A fin de determinar la variación microestructural inducida por los tratamientos realizados, se ha

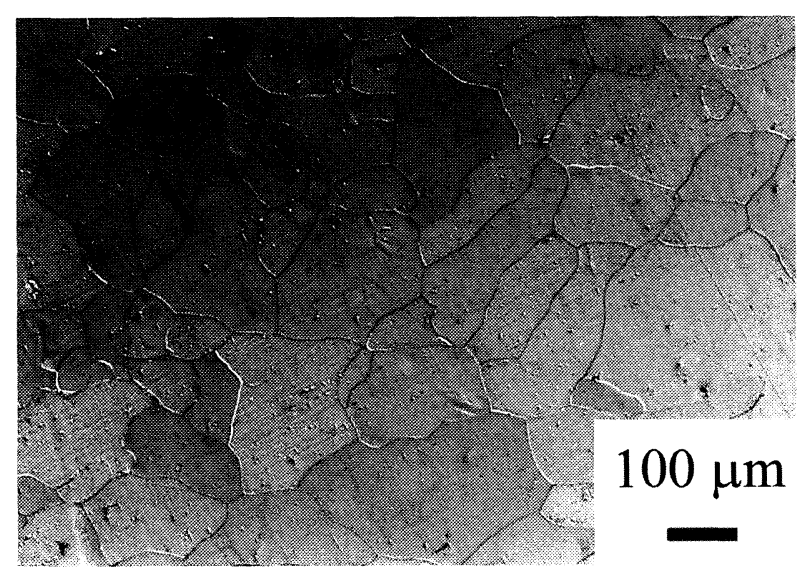

Figura 1. Microestructura del acero inoxidable superferrítico DIN 1.4575 en estado de recepción.

Figure 1. Microstructure of as received DIN 1.4575 superferritic stainless steel microstructure.
Tabla II. Condiciones de envejecimiento térmico Table II. Thermal ageing conditions

\begin{tabular}{cr}
\hline Temperatura $\left({ }^{\circ} \mathrm{C}\right)$ & \multicolumn{1}{c}{ Tiempo (h) } \\
\hline 400 & $5,24,100,500,1.000$ \\
425 & $5,24,100,500$ \\
475 & $1,5,24,100,500,1.000$ \\
500 & $1,5,8,24,100,100,500$ \\
\hline
\end{tabular}

efectuado un estudio metalográfico del material en las distintas condiciones de envejecimiento consideradas.

\section{RESULTADOS Y DISCUSIÓN}

\subsection{Efecto del envejecimiento térmico en la dureza Vickers}

Los valores proporcionados por las medidas de dureza Vickers, para cada una de las condiciones de envejecimiento consideradas, se representan en la figura 2.

A tenor de los resultados representados en el gráfico de la figura 2, se pone de manifiesto que el envejecimiento térmico produce un endurecimiento estructural progresivo, tanto más notable cuanto

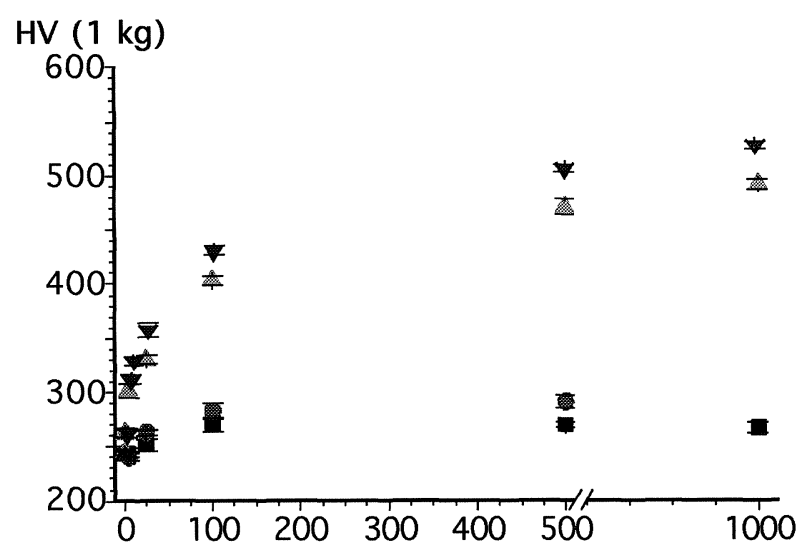

Figura 2. Variación de la dureza Vickers con el envejecimiento térmico.

Figure 2. Change in Vickers hardness during thermal ageing.

Tabla I. Composición nominal del acero superferrítico DIN 1.4575, \% en masa

Table I. DIN 1.4575 superferritic steel nominal composition, mass \%

\begin{tabular}{cccccccccc}
\hline $\mathrm{Cr}$ & $\mathrm{Ni}$ & $\mathrm{Mn}$ & $\mathrm{Mo}$ & $\mathrm{Si}$ & $\mathrm{Nb}$ & $\mathrm{N}$ & $\mathrm{C}$ & $\mathrm{S}$ & $\mathrm{P}$ \\
\hline 28,66 & 3,99 & 0,28 & 2,40 & 0,36 & 0,43 & 0,040 & 0,005 & 0,006 & 0,019 \\
\hline
\end{tabular}


más se prolonga la permanencia a la temperatura correspondiente. Tal como puede apreciarse en la figura 2, la temperatura desempeña un papel importante y, por consiguiente, el mecanismo responsable es un proceso térmicamente activado ${ }^{[7}$ y 8$]$.

La observación y cuantificación, por microscopía electrónica de transmisión, de los cambios microestructurales que ocurren durante el envejecimiento es compleja, debido a las similaridades existentes entre el poder dispersor de los átomos de hierro y de cromo. En estudios realizados en aceros inoxidables ferríticos que han sufrido descomposición espinodal, $\alpha \rightarrow \alpha$, se ha detectado que entre el 70 y el $80 \%$ del contenido inicial de cromo se concentra en los precipitados de $\alpha^{\prime}$ y, aunque la diferencia entre los parámetros de red de ambas fases es únicamente de un $0,2 \%$, la progresiva densidad de precipitados provoca la aparición de tensiones internas que se traducen en un considerable aumento de la dureza ${ }^{[2]}$. Esta transformación microestructural es fruto de la difusión del cromo en la red del hierro, de manera que puede considerarse que el envejecimiento térmico es un proceso controlado por la difusión del cro$\mathrm{mo}^{[9}{ }^{[0]}$. Si los cambios en la dureza, observados durante el envejecimiento, son producto de un proceso térmicamente activado, es posible, entonces, describir la dureza en función del tiempo y la temperatura según una ley exponencial ${ }^{[6]}$ del tipo

$$
H V_{T}(t)=H V(\infty)-\Delta H V(\infty) \exp \left(-\frac{t}{t_{c}(T)}\right)
$$

donde, $H V_{T}(t)$ representa el valor de dureza del material tras permanecer $t$ horas a la temperatura $T ; H V(\infty)$ es el valor máximo de dureza (tras un envejecimiento muy prolongado); $\Delta H V(\infty)$ representa el máximo incremento de dureza, que se alcanza entre $t=0$ y $t=\infty$. El parámetro $t_{c}(T)$ representa el tiempo característico que se precisa para alcanzar un aumento en la dureza igual a un 63,2 $\%$ del máximo valor alcanzable $(\Delta H V(\infty))$.

$$
\Delta H V_{T}\left(t_{c}\right)=\Delta H V(\infty)\left(1-\frac{1}{\mathrm{e}}\right)
$$

A partir del ajuste de ecuaciones del tipo (1) a las curvas experimentales de dureza/tiempo a las distintas temperaturas, es posible obtener los valores de $t_{c}(T)$ ya que, partiendo de la expresión (1) y tomando logaritmos, se tiene que

$$
\log \left(\frac{H V(\infty)-H V_{T}(t)}{\Delta H V(\infty)}\right)=-\frac{t}{t_{c}(T)}
$$

de manera que $t_{c}(T)$ se obtiene a partir de la pendiente de la recta (3). Este parámetro resulta característico para cada temperatura de envejecimiento y deviene un indicador del ritmo de endurecimiento asociado ${ }^{[3,6 \text { y } 11]}$. Los valores determinados para $t_{c}(T)$ se indican en la tabla III y ponen de manifiesto que el envejecimiento es muy sensible a la temperatura.

Así, con el superferrítico DIN 1.4575 y, para una temperatura de $400^{\circ} \mathrm{C}$, se requiere de un tiempo 40 veces mayor al necesario para alcanzar el mismo grado de endurecimiento a $500^{\circ} \mathrm{C}$.

\subsection{Efecto del envejecimiento térmico en la microestructura}

El estudio metalográfico realizado para el material en las distintas condiciones de envejecimiento térmico consideradas, ha revelado la formación de maclas cuando el acero superferrítico es enfriado rápidamente, desde la temperatura de envejecimiento térmico, por inmersión en agua. Se comprobó que no presentaba la formación de maclas cuando se enfriaba al aire, desde la temperatura de tratamiento.

La densidad de maclado se ha revelado tanto más notable para mayores grados de envejecimiento si bien, no se ha podido vincular directamente con el endurecimiento estructural ya que el material tratado a $400^{\circ} \mathrm{C}$, aún para períodos relativamente largos de permanencia, no ha mostrado un aumento significativo en los valores de dureza pero sí un notable incremento en la cantidad de maclas (Fig. 3).

Los tratamientos térmicos de envejecimiento realizados a las temperaturas mayores, dentro del rango intermedio considerado, han puesto de manifiesto que la formación de maclas es mucho más intensa en estas condiciones ya que, tras pocas horas de tratamiento, se observa un maclado considerable en el material (Fig 4).

Tabla III. Parámetro tc(T) para tratamientos de envejecimiento a distintas temperaturas

Table III. $t_{c}(T)$ parameter for different thermal ageing temperatures

\begin{tabular}{cc}
\hline Temperatura $\left({ }^{\circ} \mathrm{C}\right)$ & tc $(\mathrm{T})(\mathrm{h})$ \\
\hline 400 & 8.130 \\
425 & 2.438 \\
475 & 472 \\
500 & 183 \\
\hline
\end{tabular}


Formación de maclas durante el enfriamiento en aceros inoxidables superferríticos envejecidos M.N. SALÁN Y M.J. ANGLADA
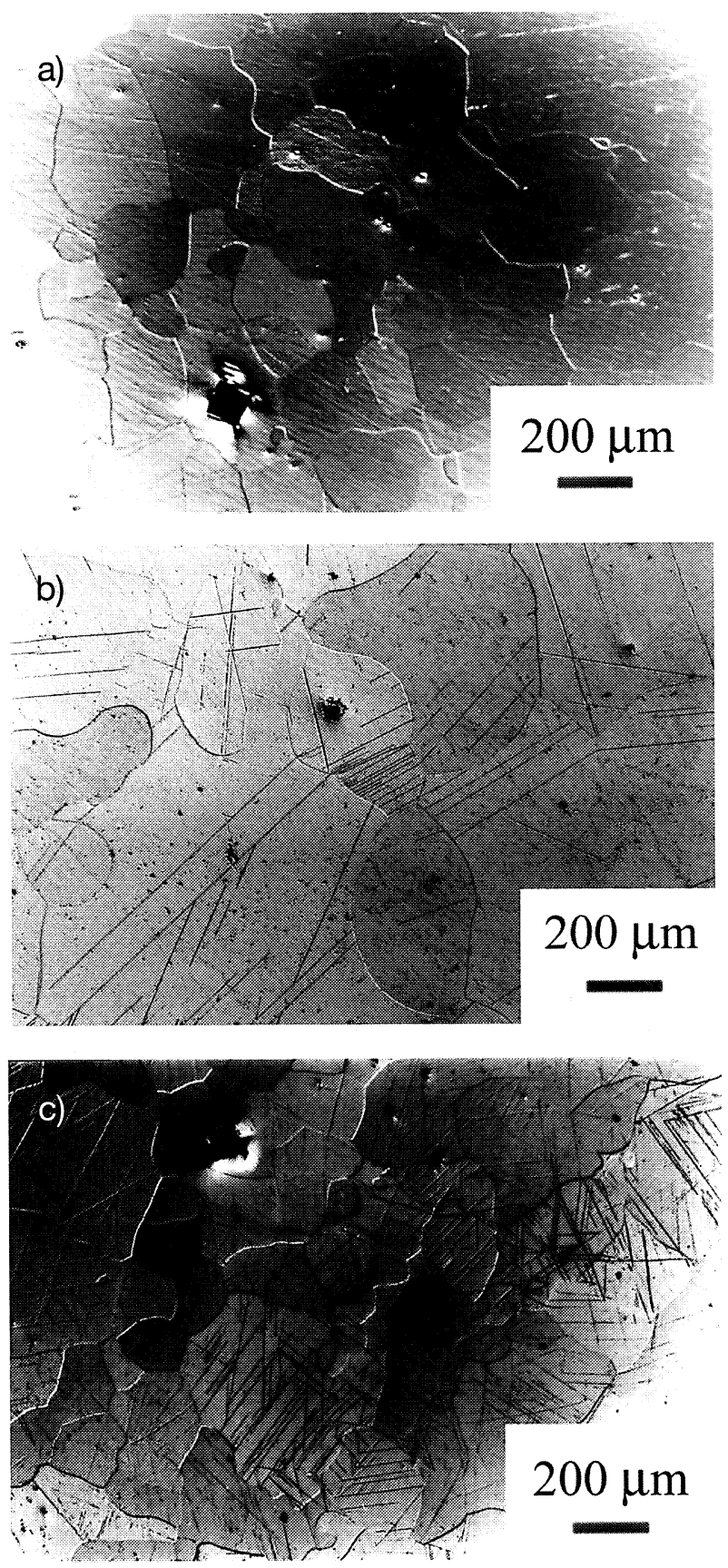

Figura 3. Microestructuras después del envejecimiento térmico a una temperatura de $400{ }^{\circ} \mathrm{C}$ durante: a) $5 \mathrm{~h}$; b) $100 \mathrm{~h}$; c) $1000 \mathrm{~h}$.

Figure 3. Microstructures after different thermal ageing conditions at $400^{\circ} \mathrm{C}$ during: a) $5 \mathrm{~h}$; b) $100 \mathrm{~h}$; c) $1000 \mathrm{~h}$.

Por otra parte, se estima que las tensiones térmicas inducidas en la superficie para enfriamientos severos ideales son superiores al límite elástico del material. Es posible, por tanto, que las tensiones térmicas reales en los enfriamientos realizados alcancen fácilmente una fracción importante del límite elástico del material.

Esta apreciación es coherente con la presencia de maclado en el acero estudiado durante la reali-
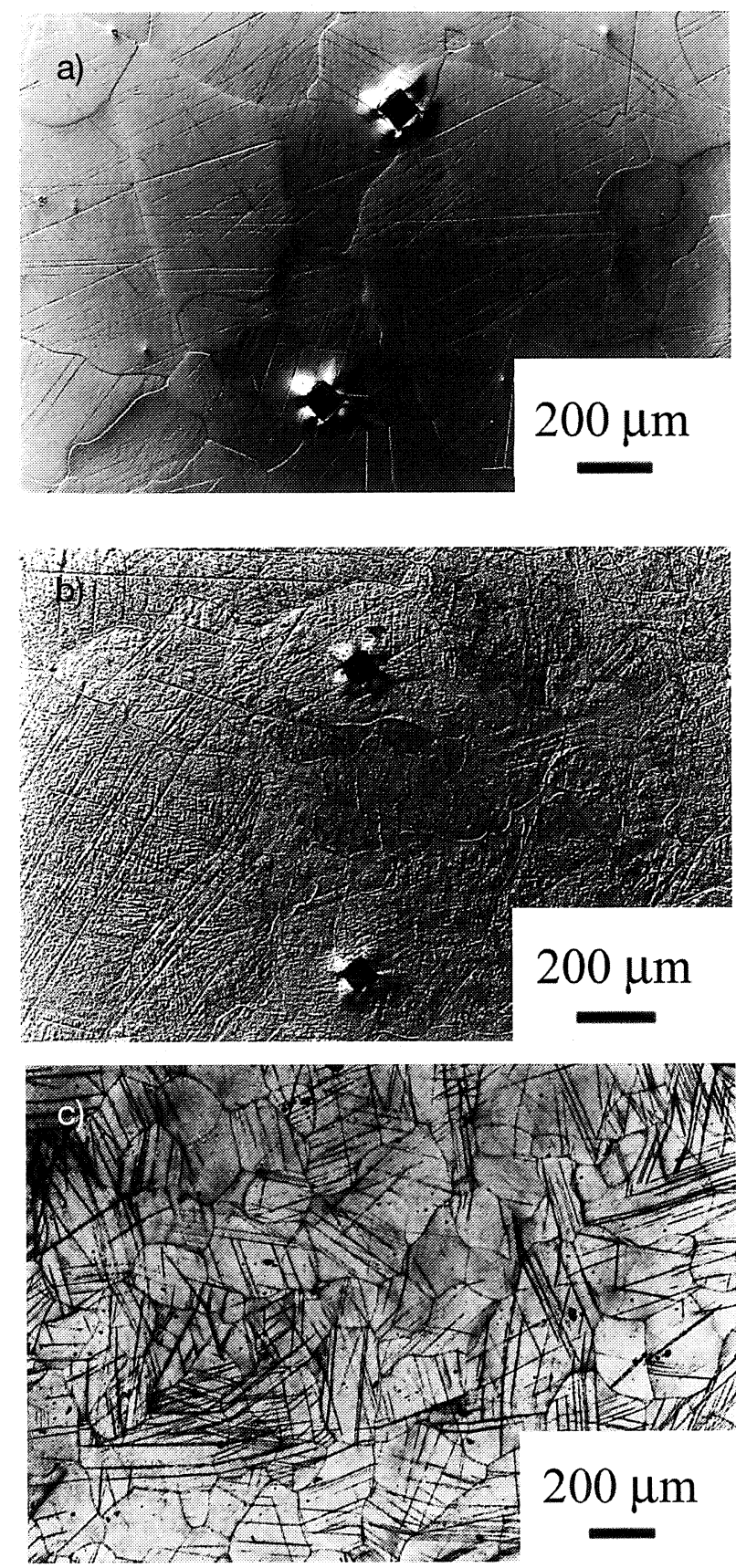

Figura 4. Microestructuras después del envejecimiento térmico a una temperatura de $475^{\circ} \mathrm{C}$ durante: a) $1 \mathrm{~h}$; b) 25 h; c) $1000 \mathrm{~h}$.

Figure 4. Microstructures after different thermal ageing conditions at $475^{\circ} \mathrm{C}$ during: a) $1 \mathrm{~h}$; b) $25 \mathrm{~h}$; c) $1000 \mathrm{~h}$.

zación de ensayos convencionales de tracción a temperatura ambiente para valores de tensión inferiores al límite elástico ${ }^{[6}$ y 12$]$.

La razón del cambio en el comportamiento debe estar relacionada con el cambio en el modo de deformación de la ferrita que induce la descomposición espinodal. De hecho, se ha observado que la distribución de dislocaciones que resulta después de la deformación del acero en estado de recocido, 
pasa a ser planar y localizada en el acero envejeci$\mathrm{do}^{[3]}$.

\section{CONCLUSIONES}

Para el superferrítico DIN 1.4575, la evolución de la dureza durante el tratamiento térmico, a temperaturas comprendidas en el rango $400-500{ }^{\circ} \mathrm{C}$, se puede describir mediante una ley exponencial asintótica, compatible con un mecanismo térmicamente activado.

Se ha puesto de manifiesto que, en el acero inoxidable superferrítico DIN 1.4575 envejecido y enfriado rápidamente por inmersión en agua desde la temperatura de tratamiento, se forman maclas en la superficie. La causa de este fenómeno puede hallarse en las tensiones térmicas inducidas por el choque térmico y por el aumento de la tendencia al maclado, provocado por el endurecimiento por descomposición espinodal.

\section{REFERENCIAS}

[1] M. KATO, Acta Metall. 29 (1981) 79-87.
[2] J. Hochmann, Aciers Inoxydables, Techniques de l'Ingénieur 1 (M 320 a), Francia, 1977, p. 11.

[3] T.J. Nichol, A. DatTa y G. Aggen, Metall. Transactions 11A (1980) 573-585.

[4] H.M. Chung y O.K. Chopra, Proc. Environmental Degradation of Materials in Nuclear Power Systems, G.J. Theus y J.R.Weeks (Eds.), 1988, pp. 359-370.

[5] A. Trautwein y W. Gysel, Stainless Steel Castings, ASTM STP 756, V.G. Behal y A.S. Melilli (Eds.) ASTM, Philadelphia, EEUU, 1982, pp.165-172.

[6] N. SAlÁn, Tesis Doctoral, Escuela Técnica Superior de Ingeniería Industrial de Barcelona, Universidad Politécnica de Catalunya, Barcelona, 2002.

[7] F.B. Pickering (Ed.), The Metallurgical Evolution of Stainless Steels, ASM, Ohio, EEUU, 1979, p. 284.

[8] J.R. DAVIS (Ed.), Specialty Handbook: Stainless Steels, ASM, Ohio, EEUU, 1994, p. 19.

[9] P.H. Pumphrey y K.N. AkHurst, Mater. Sci. Technol. 3 (1990) 211-218.

[10] O.K. ChOpRA, $18^{\text {th }}$ Water Reactor Safety Information Meeting, Rockville, EEUU, 1990, pp.22-31.

[11] J.O. Nilsson y P. Liu, Mater. Sci. Technol. 9 (1991) 853 862.

[12] M. Anglada, M. Nasarre y J.A. Planell, Scripta Metallurgica 21 (1987) 931-936. 